\section{KENTRON}

REVUE PLURIDISCIPLINAIR

DU MONDE ANTIQUE

\section{Kentron}

Revue pluridisciplinaire du monde antique

32 | 2016

Approches historiennes des images (I)

\title{
Aspetti della tradizione manoscritta di Coricio di Gaza (IV)
}

Paola D'Alessio

\section{OpenEdition}

1 Journals

\section{Edizione digitale}

URL: http://journals.openedition.org/kentron/900

DOI: $10.4000 /$ kentron.900

ISSN: 2264-1459

\section{Editore}

Presses universitaires de Caen

\section{Edizione cartacea}

Data di pubblicazione: 31 décembre 2016

Paginazione: 199-212

ISBN: 978-2-84133-840-5

ISSN: 0765-0590

Notizia bibliografica digitale

Paola D'Alessio, « Aspetti della tradizione manoscritta di Coricio di Gaza (IV) », Kentron [En ligne], 32 I

2016, mis en ligne le 10 mai 2017, consulté le 18 novembre 2020. URL : http://

journals.openedition.org/kentron/900; DOI : https://doi.org/10.4000/kentron.900

Questo documento è stato generato automaticamente il 18 novembre 2020.

\section{(c) (i) $\odot$}

Kentron is licensed under a Creative Commons Attribution-NonCommercial-NoDerivatives 3.0

International License. 


\title{
Aspetti della tradizione manoscritta di Coricio di Gaza (IV)
}

\author{
Paola D’Alessio
}

Desidero ringraziare vivamente Eugenio Amato (Université de Nantes/IUF), per i preziosi consigli elargiti durante la stesura del presente articolo. Un sincero ringraziamento va, altresì, all'amico e collega Matteo Deroma, per la sua costante disponibilità e collaborazione. Le prime tre serie si leggono, rispettivamente, in D'Alessio 2016, D'Alessio 2014, e D'Alessio 2014-2015.

Ad Eugenio Quid dulcius quam habere quicum omnia audeas sic loqui ut tecum! Qui esset tantus fructus in prosperis rebus, nisi haberes qui illis aeque ac tu ipse gauderet?

Cicerone, Lael. 22

1 La tradizione manoscritta di Coricio di Gaza si arricchisce di due nuovi manoscritti, finora sconosciuti agli editori : l'Esc. T II 9 ed il Vat.gr. 224, presentati e analizzati nella prima parte dell'articolo. La seconda parte del presente contributo verte sull'identificazione dei manoscritti che Frédéric Morel utilizzò per la sua edizione del 1606 del Patroclus di Coricio (op. XXXVIII Foerster-Richtsteig).

\section{Due codici inescussi di Coricio}

2 Le ricerche che conduco da alcuni anni sulla tradizione manoscritta di Coricio di Gaza mi hanno permesso, tra l'altro, di ampliare la recensio dei manoscritti con la scoperta di due nuovi testimoni, conservati, rispettivamente, in Spagna e nella Città del Vaticano, finora del tutto sconosciuti agli studiosi coriciani : si tratta dei manoscritti Esc. T II 9 e Vat. gr. 224.

Il primo 2 è un manoscritto cartaceo, della seconda metà del sec. XVI, formato da 329 fogli, che, ai ff. 69-2953 , risulta essere copia dell'editio princeps di Libanio ${ }^{4}$.

4 Pur contenendo il Rhetor di Coricio (op. XLII F.-R.), il manoscritto era sfuggito agli editori del retore di Gaza. Eppure, già nel catalogo di Revilla del 193655, il codice era 
stato descritto nel dettaglio e, per i ff. 113-124, era stato indicato [Choricii rhetoris oratio]

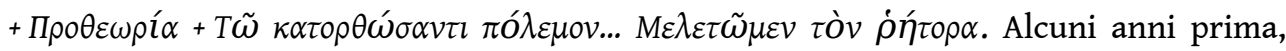
nell'edizione libaniana curata dal Foerster nel $1903^{6}$, il medesimo manoscritto era stato segnalato tra i testimoni di Libanio $^{7}$ ed era stato esplicitamente indicato come ex editione Ferrariensi descriptus, ma nessun riferimento si era fatto allora allo scritto del Gazeo.

5 È chiaro che il nuovo testimone non ha alcuna importanza ai fini della constitutio textus, essendo esso copia diretta di un'edizione a stampa. È, tuttavia, interessante il fatto che sia stata prodotta una copia di un testo stampato, il che testimonia la grande importanza che dovettero rivestire, nel corso del XVI secolo, le opere del retore di Antiochia e, dunque, molte delle opere di Coricio che a Libanio erano ascritte.

Il libro appartenne ad Antonio Agustín ${ }^{8}$, nella cui Bibliotheca manuscripta graeca risulta essere inventariato $\mathrm{al} \mathrm{n}^{\circ} 236^{\circ} \mathrm{ed}$ ivi così descritto : THEON ET LIBANIUS. Theonis Sophistae progymnasmata Rethorica, cum variis lectionibus. Libanii Sophistae exercitationes, sive declamationes; orationes quoque atque ecphrases numero XXXVIII. Incerti de prooemiis Rethoricis. Joanne Doxopatris in Aphthonii progymnasmata. Postremi duo tituli descripti sunt ex antiquo codice Physicorum ARISTOTELIS supra num. 201. Liber in charta annorum LX forma folii ${ }^{10}$.

7 Probabilmente, la copia fu realizzata proprio per Antonio Agustín, detentore di una ricca biblioteca - poi confluita in quella del monastero de El Escorial -, della quale lo stesso vescovo fornì un inventario dei codici, con una descrizione sufficientemente accurata dei 272 manoscritti greci da lui posseduti. Il manoscritto fu copiato in parte (ff. 1-158 1.7 ) da Andronico Nuccio ${ }^{11}$, in parte (ff. 297-328) ${ }^{12}$ da Andrea Darmario ${ }^{13}$, copista, quest'ultimo, di un consistente numero di manoscritti, che produceva in più esemplari destinati alla vendita.

8 L'altro testimone di Coricio, finora sconosciuto agli editori coriciani, è il Vat. gr. 224 ${ }^{14}$, un cartaceo occidentale di 305 fogli, scritto per la maggior parte nel sec. XIV ${ }^{15}$, e costituito da differenti pezzi rilegati insieme, trascritti da copisti diversi.

Sebbene segnalato nel secondo volume dell'edizione libaniana ${ }^{16}$, dove il Foerster aveva

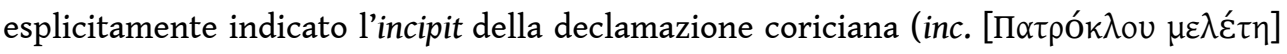
' $\Omega \mu \eta \nu \tilde{\omega} \dot{\alpha} \chi \imath \lambda \lambda \varepsilon \tilde{v})$, il manoscritto era poi sfuggito all'editore tedesco, in sede di recensio dei manoscritti di Coricio, esattamente come era sfuggita la presenza del testo del

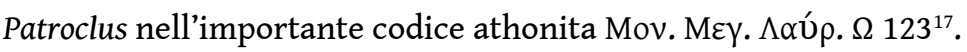

10 Realizzato con carta spessa di colore giallastro e, dunque, di cattiva qualità, il manoscritto presenta molti fogli rovinati da blatte (soprattutto nei margini) e malamente ricostruiti con strisce di carta incollate.

11 Ai fogli 229-237v , esso trasmette il Patroclus di Coricio, inserito tra le declamazioni (nell'ordine) 33 e 3 Foerster di Libanio ${ }^{18}$.

12 Nulla si sa della storia del manoscritto prima del suo ingresso in Vaticana ; la presenza nella Biblioteca Apostolica è attestata già dall'inventario dei codici redatto da Cosme de Montserrat ${ }^{19}$, sotto il fondatore della Biblioteca, Niccolò V (1397-1455), dove il libro appare descritto fra quelli matematici al $\mathrm{n}^{\circ} 350$ : Cleomedis Metheora et Luciani et Libanii nonnulla, etc. ${ }^{20}$.

13 Il codice non risulta di alcuna utilità ai fini della constitutio textus. Dalla collazione, infatti, è emerso che circa un terzo costituiscono errori propri del manoscritto o varianti, che non hanno alcun valore per la costituzione del testo del Patroclus ${ }^{21}$. Tali 
errori, omissioni e varianti, non ritrovandosi in nessun altro testimone di Coricio, sono prova del fatto che il manoscritto vaticano non ha generato alcun apografo.

Per il resto, le sue varianti sono in comune ora ai manoscritti Chalc. S. Trin. 145 e Esc. $\Psi$

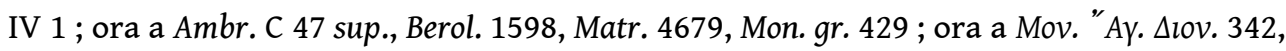
Mov. 'I $\beta .186$ e Par. gr. 2961 ; ora a Ambr. E 92 sup., Guelferb. 77, Laur. Plut. LVII.23, LVII.27 e LIX.30, Marc. gr. Z 422 e 440, Mosqu. Sinod. gr. 317, Par. gr. 3018, Par. Suppl. gr. 656, Vat. gr. 937 ; ora a Esc. $\Sigma$ I 14, Mut. II D 7, Vat. Pal. gr. 154 e 275 ; ora, ma in misura gradualmente minore, a Jer. S. Sep. 107, Laur. Plut. LVII.21, Matr. 4755, Mon. gr. 96, Vat. gr. 940 e 1392 ;

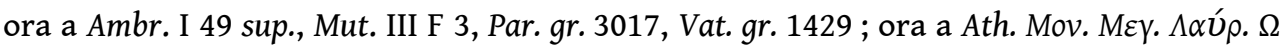
123 e Matr. 4641 ; ora a Esc. $\Psi$ IV 12 e T II 11, Neap. II E 19, Vallicell. F 14, Vat. Barb. gr. 220, Vind. Phil. gr. $82^{22}$.

Tali analogie consentono di asserire che il codice discende dal cosiddetto ramo $b^{23}$ della tradizione manoscritta del Patroclus e, vista la sua età, esso andrà posto nella parte alta dello stemma dell'op. XXXVIII F.-R. di Coricio.

In conclusione, essendo l'Esc. T II 9 copia dell'editio di Ferrara ed il Vat. gr. 224 privo di varianti interessanti, non è al loro valore ai fini della restituzione del testo di Coricio che si guarderà. Piuttosto, segnalando la presenza di questi testimoni dell'opera coriciana, si potranno ben definire i rapporti tra questi e gli altri manoscritti di Coricio e si potrà illustrare il milieu culturale di cui essi sono espressione.

\section{Le fonti manoscritte del Patroclus di Coricio nell'edizione libaniana di Fédéric Morel}

17 Nel 1606, l'officina tipografica parigina di Claude Morel diede alle stampe l'opera di Fédéric Morel, Libanii Sophistae Praeludia oratoria LXXII. Declamationes XLV et dissertationes morales ${ }^{24}$, dedicata ad Enrico IV.

18 L'edizione contiene, oltre alla Vita di Libanio tratta dalle Vite di Eunapio, un gran numero di progimnasmi ${ }^{25}$ e declamazioni ${ }^{26}$ nonché due orazioni ${ }^{27}$ del retore di Antiochia.

19 Tra le declamazioni libaniane, figurano in realtà anche il Patroclus e il Rhetor ${ }^{28} \mathrm{di}$ Coricio. Ciò non deve sorprendere perché, nei manoscritti, entrambe le opere di Coricio risultano ascritte a Libanio. Bisognerà aspettare il lavoro di Jean François Boissonade ${ }^{29}$ per vedere attribuito per la prima volta a Coricio il Patroclus (op. XXXVIII F.-R.), e quello di Richard Foerster ${ }^{30}$ perché gli sia ascritto anche il Rhetor (op. XLII F.-R.) ${ }^{31}$.

È un dato di fatto che Morel, per dare alle stampe le proprie edizioni di testi greci, si serviva di più fonti manoscritte. Così, ad esempio, per l'edizione di Dione di Prusa, pubblicata nel $1604^{32}$, Morel utilizzò tre manoscritti, cui egli accenna vagamente nella prefazione della sua opera ${ }^{33}$, ma senza ulteriori specifiche indicazioni ${ }^{34}$.

21 Analogamente, anche per l'edizione di Libanio, è pacifico che egli abbia utilizzato diversi testimoni manoscritti, la cui identificazione risulta, tuttavia, problematica. In questa sede, ci interesseremo all'individuazione dei manoscritti compulsati dall'editore parigino per gli scritti di Coricio.

Secondo Boissonade ${ }^{35}$, il codice utilizzato dal Morel sarebbe stato il Par. gr. $3017^{36}$, mentre, per Richard Foerster ${ }^{37}$, il Par. gr. $2988^{38}$. 
Un'attenta collazione del testo edito dal Morel coi testimoni contenenti il Patroclus, consente di giungere a nuove e più precise conclusioni.

Nella prefazione Lectori $\varphi \imath \lambda_{0} \lambda_{\imath} \beta \alpha v i ́ \omega$, Morel dichiara: «[...] Ferrariensem macrum,

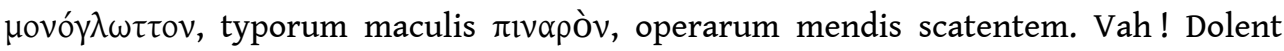
adhuc oculi ex tristi illius inamoenae, carae tamen, editionis lectione ${ }^{39}$. Lo studioso, dunque, per la sua edizione ha avuto sicuramente a disposizione l'editio princeps delle Orazioni di Libanio, apparsa a Ferrara nel 1517 per le cure di Soteriano Capsali. Nella medesima prefazione, Morel sostiene di aver ricevuto l'esemplare dall'umanista e filologo francese Etienne Turnèbe - figlio del più celebre Adrien -, ma si tratta di un mero errore, come osservato già da Foerster ${ }^{40}$, giacché l'esemplare fu inviato al Morel dal gesuita André Schott (1552-1629) ; così attesta lo stesso erudito belga nella lettera accompagnatoria del codice libaniano Brahensis Skoklosterianus IV $98^{41}$, posta ad apertura del manoscritto : in Ferrariensi editione Declamationum : [...] habet a me Morellus ${ }^{42}$. utilizzato per la sua pubblicazione, oltre all'editio Ferrariensis, anche i manoscritti Vat. Pal. gr. $282^{44}$, Vat. gr. $939^{45}$ e Par. gr. $3016^{46}$ : di questi, soltanto l'ultimo contiene pure gli scritti di Coricio.

In seguito, nei Prolegomena all'edizione coriciana ${ }^{47}$, il filologo tedesco ritiene che il Morel si sia servito, per il Patroclus ${ }^{48}$, del Par. gr. 2988, mentre afferma che non può essere provato, come invece asseriva Boissonade, che il codice utilizzato fosse il Par. gr. 3017, giacché un gran numero di lezioni accolte nel testo dal Morel non trovano riscontro in quest'ultimo manoscritto ${ }^{49}$.

Comunque sia, che Morel, per la sua edizione, abbia utilizzato più di un codice conservato nell'attuale Bibliothèque nationale de France è certo : egli, infatti, nelle sue observationes et variae curae all'edizione libaniana ${ }^{50}$, fa spesso riferimento a codici regi più 0 meno recenti.

Per individuare gli antigrafi utilizzati dallo studioso, un'attenta analisi delle lezioni del testo edito dal Morel che si discostano da quelle del Foerster evidenzia che, per la maggior parte, esse sono in comune ai manoscritti Par. gr. $2961^{51}$ e 2988. Mentre quest'ultimo testimone era stato già individuato dal Foerster ${ }^{52}$ quale fonte del Morel, il 2961 non era stato neppure preso in considerazione dal filologo tedesco : ciò senz'altro perché Foerster non ebbe la possibilità di collazionare il codice, ma solo di ispezionarlo ${ }^{53}$.

Eppure, è proprio il Par. gr. 2961 ad essere il manoscritto cui attinge più di sovente Fédéric Morel per la propria edizione. Più della metà delle varianti riportate a testo dal Morel, oppure semplicemente segnalate in margine alla propria edizione, si ritrovano identiche nel Par. gr. $2961^{54}$, così come più sporadicamente ${ }^{55}$ nell'Athon. Mov. 'I $\beta .186^{56}$, derivante dallo stesso antigrafo del Parigino $2961^{57}$. A queste, si aggiunge una serie più consistente di varianti in comune, oltre che col Parigino 2961, anche con altri testimoni $\operatorname{vari}^{58}$.

31 Che il Morel abbia utilizzato il Par. gr. 2961, lo testimoniano pure le indicazioni lessicali che l'autore fornisce al lettore ${ }^{59}$ nella prefazione alla sua opera : tutte le varianti che egli dichiara trovare "in recentiore mnsc.", infatti, si riscontrano identiche nel Parigino $^{60}$.

Foerster, invece, avendo notato che la maggior parte delle varianti riportate a testo dal Morel si ritrovano identiche nel Parigino 2988, aveva pensato che fosse proprio questo 
l'antigrafo utilizzato dal filologo francese. Lo studioso tedesco, in realtà, non avendo collazionato il Parigino 2961, non aveva potuto appurare che le medesime varianti ritornano identiche anche in quest'ultimo manoscritto. È sì vero che alcune in comune al testo del Morel e al Par. gr. $2988^{61}$ non si riscontrano nel Par. gr.. 2961, però tale osservazione consente esclusivamente di affermare che l'editore francese utilizza più manoscritti e, pur prediligendone uno in particolare ${ }^{62}$, a volte accoglie nel testo anche lezioni presentate da altri testimoni.

Quanto poi all'ipotesi di Boissonade, secondo cui il codice utilizzato dal Morel sarebbe stato il Par. gr. $3017^{63}$, essa è senz'altro da escludere : il manoscritto, infatti, fu donato dall'arcivescovo di Reims, Charles-Maurice Le Tellier, alla Biblioteca Regia parigina solo verso il $1700^{64}$.

Le analisi delle varianti portano ad affermare che anche un altro fu probabilmente il manoscritto compulsato da Morel per la propria edizione: il Par. gr. $3018^{65}$. Talune varianti proprie a questo testimone, ivi comprese aggiunte ed omissioni, si ritrovano nel testo edito dal Morel ${ }^{66}$. In verità, le medesime varianti si riscontrano pure nel Par. Suppl. gr. $656^{67}$, che, stando agli esiti delle mie collazioni, è copia diretta del Parigino $3018^{68}$. È da escludere, ad ogni modo, una dipendenza del Morel dal Suppl. gr. 656, in quanto esso fu portato a Parigi da Minoïde Mynas (1790-1860) ${ }^{69}$ non prima degli anni $1840-1855^{70}$.

Non è, infine, neppure da escludere, a riprova del fatto che il Morel abbia compulsato per la propria edizione diversi manoscritti, l'utilizzo del Par. gr. 3016, il quale condivide la maggior parte delle varianti esibite dai Parigini greci 2961, 2988 e 3018 e ritenute a testo o semplicemente segnalate da Morel. L'unica lezione in comune soltanto al

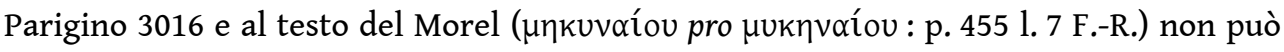
essere considerata prova del fatto che il 3016 sia stato tra le fonti manoscritte del Morel. Per Foerster, tuttavia, il manoscritto figurerebbe con certezza tra i testimoni utilizzati da Morel per l'edizione di Libanio $^{71}$.

In conclusione, è da ritenere che Fédéric Morel, per la propria edizione del Patroclus coriciano, abbia avuto la possibilità di collazionare più manoscritti parigini di quanti finora indicati dagli studiosi. In particolare, i codici cui Morel attinge la maggior parte delle lezioni per il Patroclus sono da considerare, in ordine di importanza, i Parigini greci 2961, 2988, 3016 e 3018, il primo dei quali così come il terzo mai finora individuati dalla critica.

\section{BIBLIOGRAFIA}

AMATO E. (2011), Xenophontis imitator fidelissimus. Studi su tradizione e fortuna erudite di Dione Crisostomo tra XVI e XIX secolo, Alessandria, Edizioni dell'Orso (Hellenica ; 40).

AMATO E., THÉVENET L., VENTRELla G. (edd.) (2014), Discorso pubblico e declamazione scolastica a Gaza nella tarda antichità : Coricio di Gaza e la sua opera (Atti della giornata di studio, Nantes, 6 giugno 2014), Bari, Edizioni di Pagina (Due punti ; 38). 
AMATO E., CORCELLA A., LAURITZEN D. (edd.) (2016), L'École de Gaza : espace littéraire et identité culturelle dans l'Antiquité tardive (Actes du colloque international, Paris, Collège de France, 23-25 mai 2013), Leuven, Peeters (Orientalia Lovaniensia Analecta - Bibliothèque de Byzantion) (in c.d.p.).

BIANCONI D. (2005), Tessalonica nell'età dei Paleologi. Le pratiche intellettuali nel riflesso della cultura scritta, Paris, Centre d'études byzantines, néo-helléniques et sud-est européennes de l'École des Hautes Études en Sciences Sociales.

BOISSONADE J.F. (1846), Choricii Gazaei orationes, declamationes, fragmenta. Insunt ineditae orationes duae, Parisiis, Dumont.

CARLuCCI G. (2012), I Prolegomena di André Schott alla Biblioteca di Fozio, Bari, Edizioni Dedalo (Paradosis).

DAIN A. (1957), « Les enquêtes de Minoide Mynas dans les bibliothèques grecques d'Asie Mineure (1845) », in Actes du Xe congrès international d'études byzantines (Istanbul, 15-21 novembre 1955), Istanbul, Publication du Comité d'organisation du $\mathrm{X}^{\mathrm{e}}$ congrès international d'études byzantines, p. 263-265.

D’ALESSIO P. (2014), « Aspetti della tradizione manoscritta di Coricio di Gaza (II) », in Amato et al. 2014, p. 232-266.

D’ALESSIO P. (2014-2015), « Aspetti della tradizione manoscritta di Coricio di Gaza (III) », RET, 4, p. 155-215.

D’ALESSIO P. (2016), « Aspetti della tradizione manoscritta di Coricio di Gaza (I) », in Amato et al. 2016, p. 473-520.

DE MARIA S., PARADA LóPEZ DE CORSELAS M. (2014), « Antonio Agustín, Bologna e l'antiquaria del Cinquecento ", in El Imperio y las Hispanias de Trajano a Carlo V. Clasicismo y poder en el arte españolL'Impero e le Hispaniae da Traiano a Carlo V. Classicismo e potere nell'arte spagnola, S. De Maria, M. Parada Lopez de Corselas (edd.), Bologna, Bononia University Press, p. 331-355.

DEVREESSE R. (1965), Le fonds grec de la Bibliothèque Vaticane des origines à Paul V, Città del Vaticano, Biblioteca Apostolica Vaticana.

FOERSTER R. (1903), Libanii opera, I. 1, Orationes I-V, Lipsiae, in aedibus B.G. Teubneri (BT) (rist. Hildesheim, G. Olms, 1963).

FOERSTER R. (1904), Libanii opera, II, Orationes XII-XXV, Lipsiae, in aedibus B.G. Teubneri (BT) (rist. Hildesheim, G. Olms, 1963).

FOERSTER R. (1909), Libanii opera, V, Declamationes I-XII, Lipsiae, in aedibus B.G. Teubneri (BT) (rist. Hildesheim, G. Olms, 1963).

FOERSTER R. (1915), Libanii opera, VIII, Progymnasmata. Argumenta orationum Demosthenicarum, Lipsiae, in aedibus B.G. Teubneri (BT) (rist. Hildesheim, G. Olms, 1963).

FOERSTER R. (1927), Libanii opera, IX, Libanii qui feruntur Characteres epistolici. Prolegomena ad epistulas, impr. curavit E. Richtsteig, Lipsiae, in aedibus B.G. Teubneri (BT) (rist. Hildesheim, G. Olms, 1963).

FOERSTER R., RICHTSTEIG E. (1929), Choricii Gazaei Opera, recensuit R. Foerster, editionem confecit E. Richtsteig, Lipsiae, in aedibus B.G. Teubneri (BT) (rist. Stuttgart 1972).

GAMILLSCHEG E., HARLFINGER D., HUNGER H. (edd.) (1981-1997), Repertorium der griechischen Kopisten 800-1600, 1. Teil : Grossbritannien, 1981 ; 2. Teil : Handschriften aus Bibliotheken Frankreichs und Nachträge zu den Bibliotheken Grossbritanniens, 1989 ; 3. Teil (unter Mitarbeit von P. Eleuteri) : 
Handschriften aus Bibliotheken Roms mit dem Vatikan, 1997, Wien, Verlag der Oesterreichischen Akademie der Wissenschaften (Veröffentlichungen der Kommission für Byzantinistik ; 3 / 1-3). GRAUX C. (1880), Essai sur les origines du fonds grec de l'Escurial. Épisode de l'histoire de la renaissance des lettres en Espagne, Paris, F. Vieweg.

GRAUX C. (1889), Notices sommaires des manuscrits grecs de Suède. Mises en ordre et complétées par A. Martin, Paris, Ernest Leroux (Extrait des Archives des Missions scientifiques et littéraires, $3^{\mathrm{e}}$ série ; XV).

IRIGoIN J. (1958), « Les filigranes de Fabriano (noms de papetiers) dans les manuscrits grecs du début du XIVe siècle », Scriptorium, 12, p. 44-50.

IRIGoIN J. (1999), « Une série de filigranes remarquable : les noms de papetiers de Fabriano (début du XIV siècle)», Bibliologia, 19, p. 137-147.

JACKSON D.F. (2006), « The Greek Manuscripts of John Moore and Etienne Baluze », Codices manuscripti, 56-57, p. 29-42.

LAMBRos S.P. (1900), Catalogue of the Greek Manuscripts on Mount Athos, vol. II, Cambridge, Cambridge University Press.

LENZ F.W., BEHR C.A. (1976), P. Aelii Aristidis opera quae exstant omnia. Volumen primum orationes IXVI complectens. Orationes I et V-XVI edidit Fridericus Waltharius Lenz, praefationem conscripsit et orationes II, III, IV edidit Carolus Allison Behr, Lugduni Batavorum, E.J. Brill. MARTIN J., PETIT P. (1979), Libanios, Discours, I, Autobiographie, Paris, Les Belles Lettres (CUF ; 256). MAYER M. (1997), « Towards a History of the Library of Antonio Agustín », JWC, 60, p. 261-272. MAZZUCCHI C.M., PASINI C. (2004), Nuove ricerche sui manoscritti greci dell'Ambrosiana (Atti del Convegno, Milano, 5-6 giugno 2003), Milano, Vita e Pensiero (Biblioteca erudita).

MERCATI G., FRANCHI DE' CAVALIERI P. (1923), Bibliothecae Apostolicae Vaticanae codices manu scripti recensiti [...]. Codices Vaticani Graeci, I, Codices 1-329, Romae, Typis Polyglottis Vaticanis (Cataloghi ed inventari di manoscritti).

MILLER E. (1848), Catalogue des manuscrits grecs de la Bibliothèque de l'Escurial, Paris, Imprimerie nationale (rist. anast. Amsterdam, Adolf M. Hakkert, 1966).

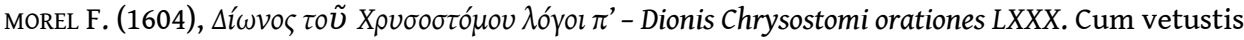
codd. mss. Reg. Bibliothecae, sedulo collatae, eorumque ope ab innumeris mendis liberatae, restitutae, auctae. Photii excerptis Synesiique censura illustratae. Ex interpretatione Thomae Nageorgi, accurate recognita, recentata \& emendata Fed. Morelli [...] opera. Cum Is. Casauboni Diatriba, et eiusdem Morelli Scholiis, Animadversionibus et Coniectaneis. Accessit rerum et verborum Index locupletissimus, Lutetiae, Ex officina Typographica Claudii Morelli, via Iacobaea, ad insigne Fontis.

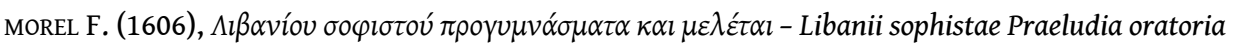
LXXII. Declamationes XLV et dissertationes morales, Federicus Morellus [...] e mss. maxime Reg. Bibliothecae nunc primum edidit : idemque Latine vertit. Adiectae sunt notae et variae lectiones cum duplici Indice locupletissimo. [...], Parisiis, Ex officina Typographica Claudii Morelli, via Iacobaea, ad insigne Fontis.

MÜNTZ E., FABRE P. (1887), La Bibliothèque du Vatican au XVe siècle d'après des documents inédits. Contributions pour servir à l'histoire de l'humanisme, Paris, Ernest Thorin (Bibliothèque des Écoles françaises d'Athènes et de Rome ; 48) (rist. Amsterdam, Gérard Th. Van Heusden, 1970). 
OMONT H. (1883), Inventaire sommaire des manuscrits du Supplément grec de la Bibliothèque nationale, Paris, A. Picard.

OMONT H. (1888), Inventaire sommaire des manuscrits grecs de la Bibliothèque nationale, Troisième partie : Ancien fonds grec, belles-lettres. Coislin - Supplément, Paris et départements, Paris, A. Picard.

OMONT H. (1916), « Minoïde Mynas et ses missions en Orient (1840-1855) », Mémoires de l'Académie des Inscriptions et Belles-Lettres, 40, p. 337-422.

PIETSCH G. (1910), De Choricio Patrocli declamationis auctore (Diss. inaug.), Vratislaviae, M. \& H. Marcus (Breslauer philologische Abhandlungen ; 42).

REVILLA A. (1936), Catálogo de los Códices Griegos de la Biblioteca de El Escorial, I, codd. 1-178, Madrid, Imprenta Helénica.

STEVENSON H. (1885), Codices manuscripti Palatini Graeci Bibliothecae Vaticanae descripti [...], Romae, ex Typographeo Vaticano.

VOGEL M., GARDTHAUSEN V. (1909), Die griechischen Schreiber des Mittelalters und der Renaissance, Leipzig, Otto Harrassowitz (Beiheft zum Zentralblatt für Bibliothekswesen ; 33).

\section{NOTE}

2. Per la descrizione del manoscritto, ved. Miller 1848, 127 ; Revilla 1936, 474-479.

3. Tale il contenuto dei fogli, che riproduce, esattamente nello stesso ordine, quanto risulta nell'edizione del 1517 di Libanio a cura di S. Capsali (vd. n. 4) : Libanio, decl. 46, 22, 42, 31, 7, 8, 37, 38 Foerster ; Coricio, op. XLII F.-R. ; Libanio, decl. 25, 24, 48, 47, 45, 34, 12, 40, 44, 26, 29, 4 Foerster ; or. 1, 3, 5, 6, 14, 15, 61, 12, 56 Foerster ; prog. IX.7, VIII.8, X. 5, XII.3, 4, 7, 5, 6, 1, 2 Foerster ; or. 7, 8 Foerster.

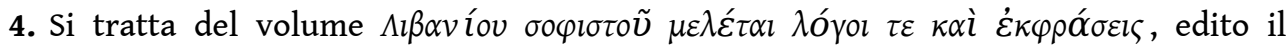
4 agosto 1517 nella tipografia di Giovanni Mazzocchi, a Ferrara, per le cure di Soteriano Capsali.

5. Revilla 1936, 476.

6. Ved. Foerster 1903, $67 ; 69$.

7. Nessun riferimento è fatto, invece, al Rhetor di Coricio.

8. Su Antonio Agustín (1517-1586), vescovo di Tarragona, filologo e bibliofilo, la cui ricchissima biblioteca conflù̀ all'Escorial, ved. De Maria \& Parada Lopez de Corselas 2014 ; Mayer 1997 ; Revilla 1936, XCV-XCVIII ; Graux 1880, 13-17 ; 280-299 ; Miller 1848, IX-XIV.

9. Ved. Antonii Augustini archiepiscopi Tarraconensis Opera omnia [...]. Volumen septimum, Lucae, Typis Josephi Rocchii, 1772, p. 62.

10. Il manoscritto, oltre alle opere di Libanio e di Coricio trascritte dall'edizione di Ferrara, contiene anche i Progimnasmi di Teone (ff. 1-30) e quelli di Libanio (ff. $30^{\mathrm{v}}-63^{\mathrm{v}}$ ), così come scritti retorici di Giovanni Dossapatre (ff. 297-329). Anche per la prima parte, il manoscritto risulta essere copia di un'edizione a stampa: i Progimnasmi di Teone,

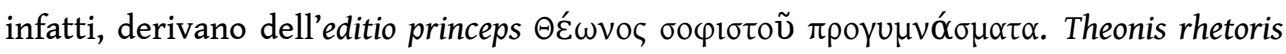
De modo declamandi libellus, curata da Angelo Barbato e pubblicata a Roma nel 1520 (ved. Foerster 1915, 8-9). 
11. Su Andronico Nuccio di Corfù, attivo prevalentemente a Venezia, dove trascrisse manoscritti per l'ambasciatore spagnolo Diego Hurtado de Mendoza, ved. Vogel \& Gardthausen, 1909, 31 ; Gamillscheg et al. 1981, $\mathrm{n}^{\circ} 20 ; 1989, \mathrm{n}^{\circ} 27 ; 1997, \mathrm{n}^{\circ} 32$.

12. Resta non ancora identificata la mano che ha copiato i f. $158^{\mathrm{v}} 1.8$ - f. 295.

13. Ved. Vogel \& Gardthausen 1909, 23 ; Gamillscheg et al. 1981, 13 ; 1989, 21 ; 1997, 22. Su Andrea Darmario di Epidauro, che giocò un ruolo importante nella formazione della biblioteca greca di Agustín, ved. Graux 1880, 287-297; sul rapporto tra Agustín e Darmario, cfr. Mayer 1997, 266.

14. Per la descrizione del manoscritto, ved. Foerster 1904, 194-195 ; Mercati \& Franchi de' Cavalieri 1923, 292-295.

15. Al sec. XIV rimandano le differenti mani che hanno trascritto i fascicoli componenti il manoscritto ; la maggior parte di esse, compresa quella del copista che ha vergato la sezione più consistente del manoscritto (ff. 55-241), contenente anche il Patroclus, risente di evidenti influssi di Fettaugen-Mode, come mostrano le pance delle lettere $\alpha, \sigma$, o, $\omega, \varphi, \delta$, molto pronunciate. Allo stesso periodo di realizzazione rinviano anche le filigrane. Per la loro identificazione, ved. Bianconi 2005,$41 ; 148-149 ; 153 ; 250$; tavv. 13-15 ; Irigoin 1999 ; Irigoin 1958, 45-46 ; Mercati \& Franchi de' Cavalieri 1923, 295.

16. Ved. Foerster 1904, 194-195.

17. Ved. D’Alessio 2016, 485 e n. 53.

18. Tale il contenuto del manoscritto : Cleomede, De motu circulari corporum caelestium ; riproduzioni di figure geometriche con didascalie ; Giuseppe Racendita, Synopsis artis

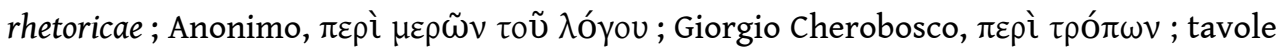
di anni; Luciano, Somnium sive vita Luciani; De parasito sive artem esse parasiticam; Adversus indoctum et libros multos ementem; Calumniae non temere credendum ; Symposium ; Timon; Icaromenippus; Vocalium iudicium; Muscae encomium; Harmonides; Phalaris; Zeuxis; Pro lapsu inter salutandum; Apologia; De mercede conductis potentium familiaribus; Elio Aristide, or. 16, 34, 18, 22, 19 Keil ; Libanio, decl. 5 Foerster ; or. 64 e 17 Foerster ; Elio Aristide, or. 31 Keil ; Libanio, decl. 46 e 33 Foerster ; Ps.-Luciano, Asinus ; Anonimo, testi di grammatica e metrica ; Manuele Moscopulo, $\dot{\alpha} \rho \imath \theta \mu \grave{\varsigma} \varsigma \pi \delta \delta \tilde{\omega} v$; Anonimo, 99 versi

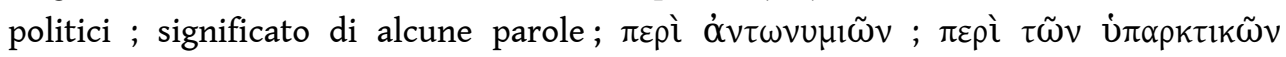

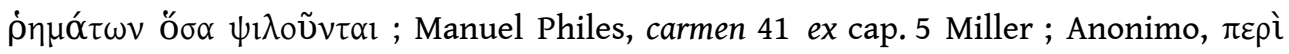

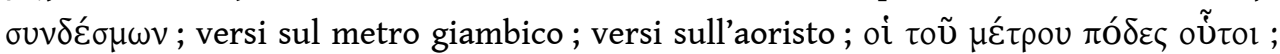
Libanio, decl. 42 e 33 Foerster ; Coricio, op. XXXVIII F.-R.; Libanio, decl. 3 e 22 Foerster ;

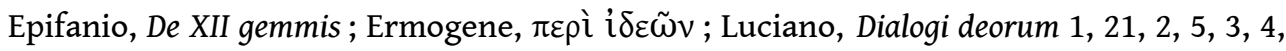
$6,7,9,8,10-15,17,16,18-20,22-24$; Dialogi marini 1-15; Dialogi mortuorum 1-30; Menippus sive necyomantia ; Charon sive contemplantes; Reviviscentes sive piscator ; Vitarum auctio ; Cataplus sive tyrannus ; Dialogi deorum 24-25.

19. Su di lui, ved. Devreesse 1965, 10.

20. Ved. Müntz \& Fabre 1887, 339, e Devreesse 1965, 36.

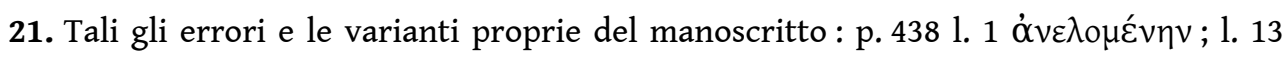

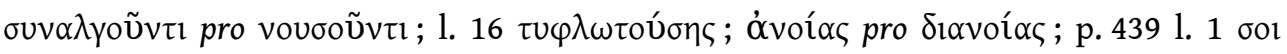

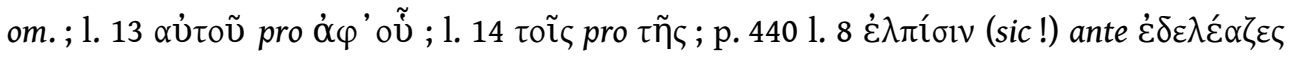

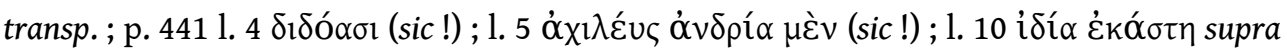

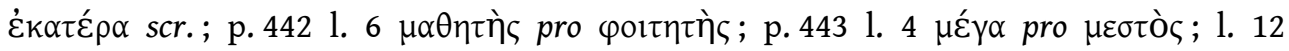

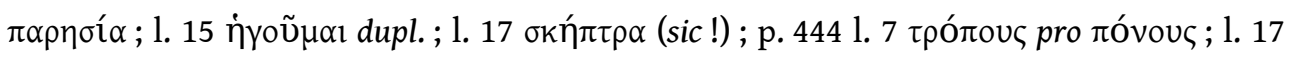




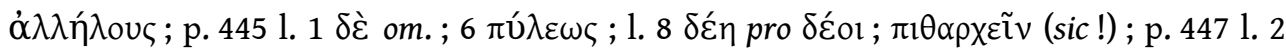

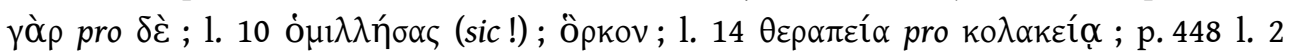

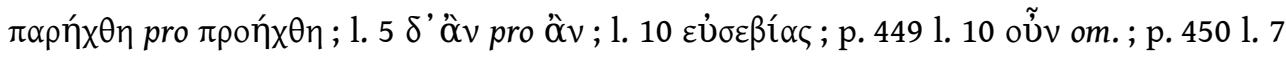

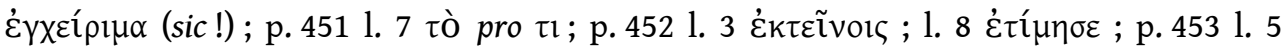

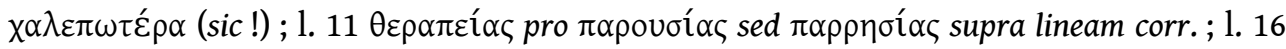

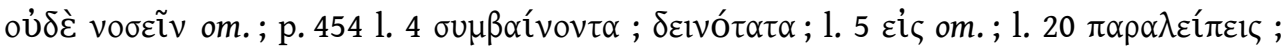

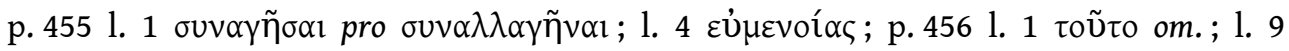

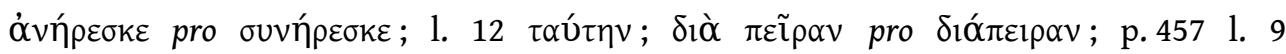

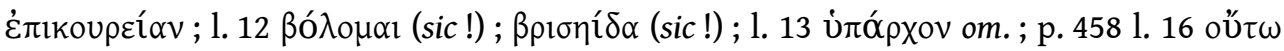

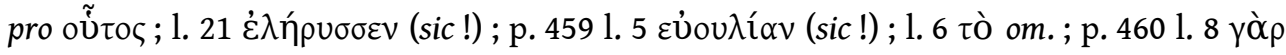

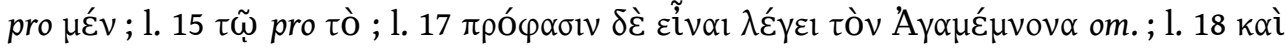

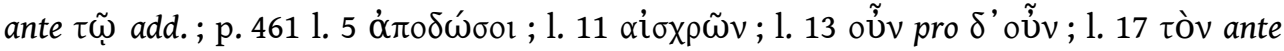

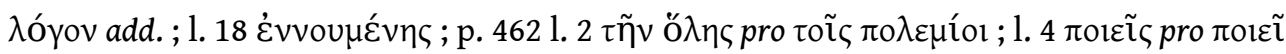

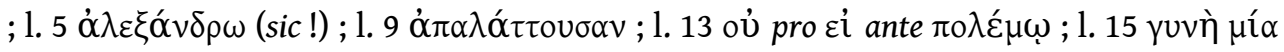

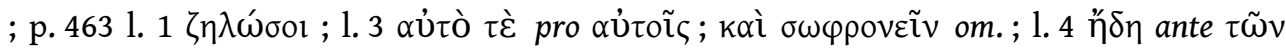

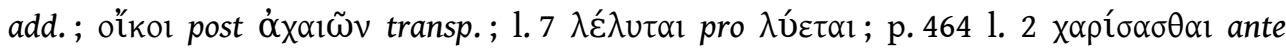

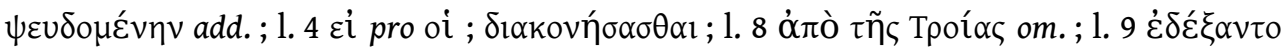

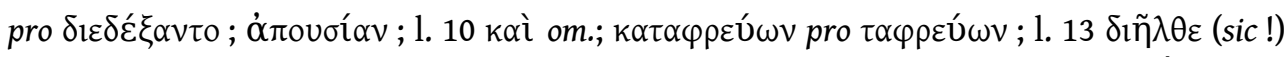

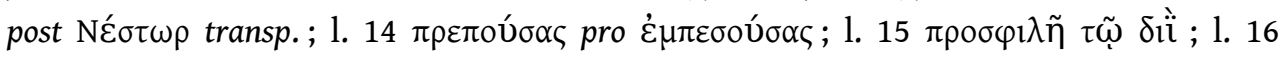

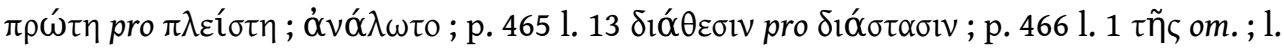

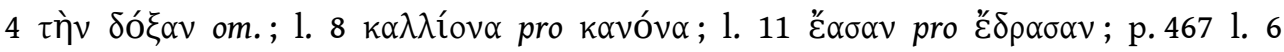

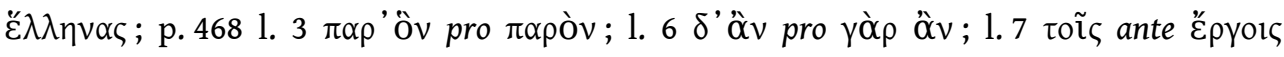

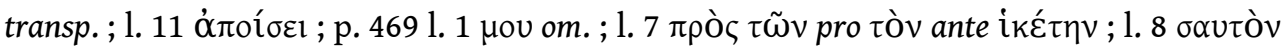

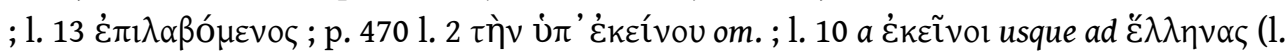

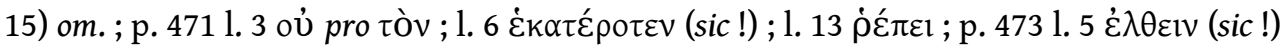

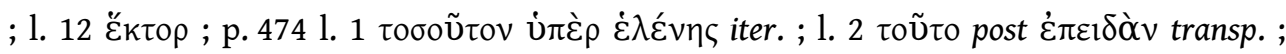

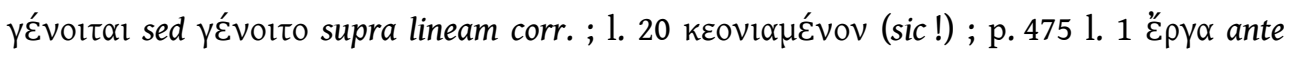

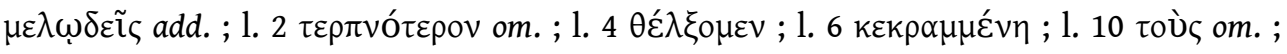

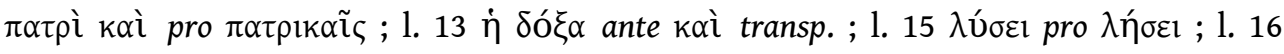

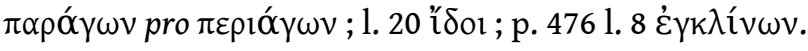

22. Per l'individuazione delle famiglie dei manoscritti del Patroclus di Coricio, ved. D'Alessio 2016, 485-486.

23. Ved. lo stemma codicum parziale pubblicato in D'Alessio 2016, 490.

24. Cfr. Morel 1606.

25. Tali, nell'ordine, i progimnasmi : I.1-3 ; II.1-3 ; IV.1-2 ; V.1 ; VI.1-2 ; VII.1-5 ; VIII.2-3, 1, 4-5, 7, 6, 8 ; IX.1-8 ; X.4, 1-3, 5 ; XI.1-2 , 14, 8-9, 11, 19, 21, 3, 12-13, 23-25, 17, 15, 4, 16, 7, 5-6, 10, 20, 18, 22 ; XII.1-2, 6-7, 5, 3, 4 ; XIII.1 Foerster.

26. Le declamazioni sono le seguenti : Libanio decl. 3-5 Foerster ; Coricio op. XXXVIII F.R. ; Libanio decl. 6, 26, 28, 30, 12, 31, 29, 35, 19, 22, 21, 23, 18, 11, 13, 9, 10, 7, 8, 24, 25, 14, 42, 44, 1, 45 Foerster ; Coricio op. XLII F.-R. ; Libanio decl. 37-40, 27, 33, 46, 43, 47, 48, 36, 41,49 Foerster.

27. Le due orazioni sono, nell'ordine : or. 8 ; 6 Foerster.

28. Morel 1606, 256-279 e 700-726. 
29. Ved. Boissonade 1846, 239 n. 1. La paternità coriciana del Patroclus viene confermata da Pietsch 1910, 8, a seguito dell'analisi stilistico-lessicale della declamazione. Per uno status quaestionis delle edizioni di Coricio, ved. D'Alessio 2016, 475-481.

30. Cfr. Foerster \& Richtsteig 1929, XXIV (altrove abbreviato F.-R.).

31. Ved. D'Alessio 2016, 476 ; 478-479.

32. Ved. Morel 1604.

33. Cfr. Morel $1604, \mathrm{f} . \mathrm{a}_{\mathrm{III}}$ : "Certe non sine Palladio seu Hermathena Bibliothecae regiae, in cuius peplo Mineruali inuolutum volumen membranarum Dionaearum euoluentes, Venetae editionis maculas plerasque eluimus, \& lacunas etiam aliquot repleuimus: ut magna pridem ex parte feceramus, eodem codice Veneto cum veteribus membranis, ex Bibliotheca Patrum Societatis IESV, diligenter collato; et cum alio MS. cuius copiam benigne nobis fecerat, vir illustriss. vetustate familiae armisque \& litteris ornatiss. I. FOXIVS Carmanii Comes $\mu \alpha \kappa \alpha$ ítn ".

34. Si tratta dei manoscritti Par. gr. 2959 (A), Leid. B.P. gr. 2C (M) e, probabilmente, Par. gr. 3009 (C). Ved. Amato 2011, 70 e nn. 6-9.

35. Boissonade 1846, 259 n. 8.

36. Per la descrizione del manoscritto (sec. XIV / XV), ved. D’Alessio 2016, 513-516.

37. Foerster \& Richsteig 1929, XXV, n. 1.

38. Per la descrizione del manoscritto, del sec. XIV, ved. Foerster 1909, 182-183 ; Omont, $1888,80$.

39. Ved. Morel 1606, f. $\mathrm{a}_{\mathrm{VII}}$.

40. Ved. Foerster 1903, 68 n. 3.

41. Il manoscritto, del sec. XIII, è attualmente conservato nella Skoklostersamlingen del Riksarkivet di Stoccolma e reca la segnatura E 8996. Per la descrizione, ved. Carlucci 2012, 293 n. 70 ; Foerster 1927, 109-112 ; Graux 1889, 77-78.

42. L'esemplare di Schott fu utilizzato anche da Johann Jakob Reiske e da Richard Foerster. Ved. Carlucci 2012, 298 n. 82.

43. Ved. Foerster 1903, 67-68.

44. Il manoscritto, realizzato nei primi anni del sec. XIV a Tessalonica da un unico scriba, è presentato in Martin \& Petit 1979, 71-72 ; Foerster 1903, 20-23 ; Stevenson 1885, 158-159. Per una bibliografia più dettagliata, ved. Mazzucchi \& Pasini 2004, 27-28 n. 46.

45. Per la descrizione del manoscritto, del sec. XIV / XV, ved. Portius, Inventarium Graecorum codicum manu scriptorum a Laurentio Portio Scriptore Graeco descriptum, II, p. 534-538.

46. Per la descrizione del manoscritto, un cartaceo del sec. XIV, ved. Jackson 2006, 34 ; 37 ; Martin \& Petit 1979, 81-82 ; Foerster \& Richtsteig 1929, XXIV ; Foerster 1903, 53-59.

47. Foerster \& Richtsteig 1929, XXIV-XXV.

48. Ibid.

49. Ibid., XXV n. 1

50. Ved. Morel 1606.

51. Per la descrizione del manoscritto, ascrivibile al sec. XV / XVI, ved. Lenz \& Behr 1976, XXIX ; Foerster \& Richtsteig 1929, XXII ; XXVII ; Foerster 1904, 188-190. 
52. Foerster 1909, 198, e Foerster \& Richtsteig 1929, XXIII-XXIV.

\section{Foerster \& Richtsteig 1929, XXII.}

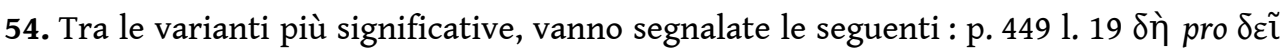

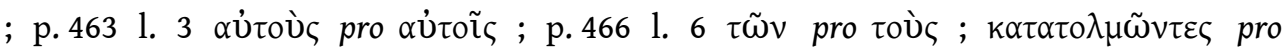

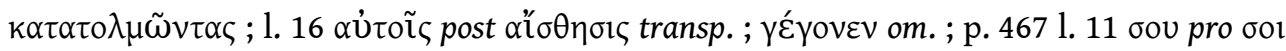

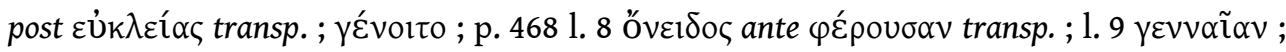
p. $4701.6 \tau \tilde{\omega} v$ ante $\theta \varepsilon \tilde{\omega} v$ add. ; p. 470 l. $11 \mu \alpha \rho \tau u \rho \circ \tilde{v} \sigma$ pro $\kappa \alpha \rho \tau \varepsilon \rho o \tilde{\sigma} \sigma$; p. 4721.6 ǐ $\sigma \omega \varsigma$

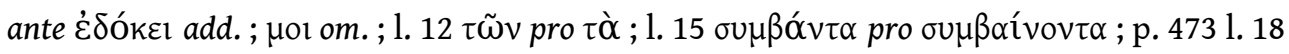

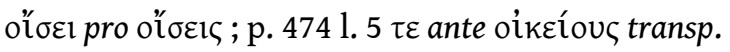

55. Di seguito le varianti in comune ai manoscritti Par. gr. 2961 e Athon. Mov. 'I $\beta .186$ :

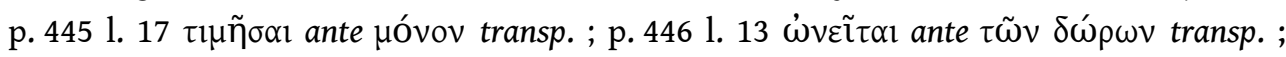

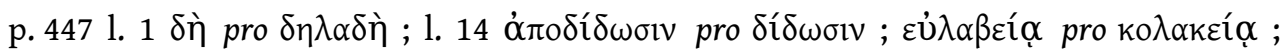

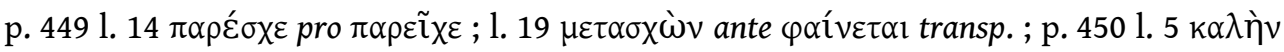

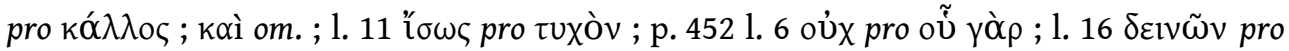

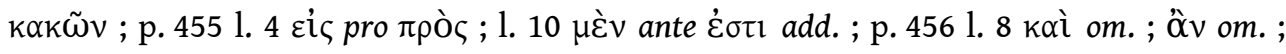

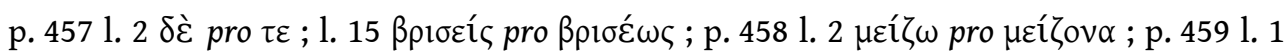

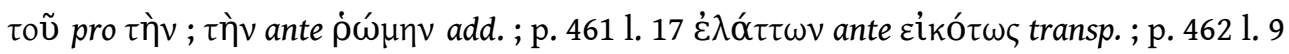

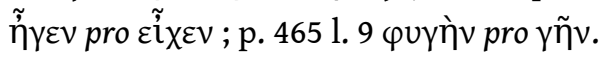

56. Per la descrizione del manoscritto, dei secc. XV-XVI, si rimanda a Foerster 1904, 228-229; Lambros 1900, 50-51.

57. Sul comune antigrafo dei due manoscritti, mi permetto di rinviare ad un mio prossimo lavoro.

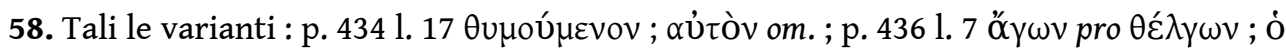

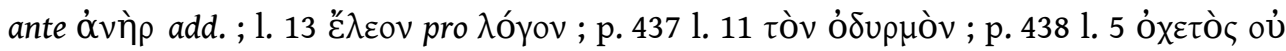

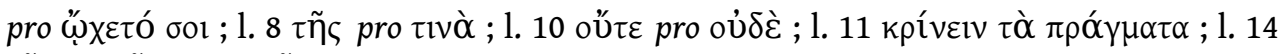

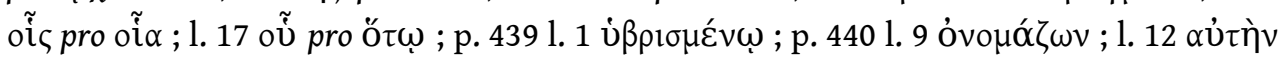

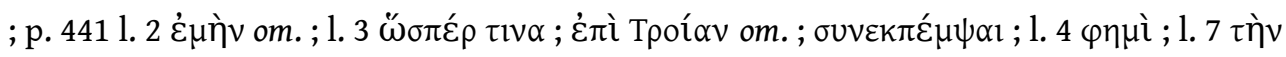

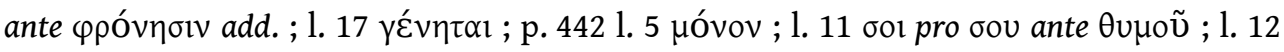

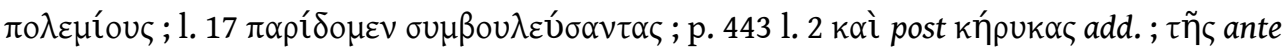

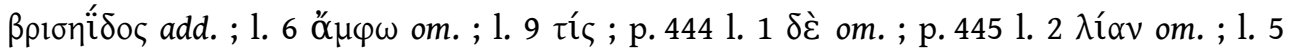

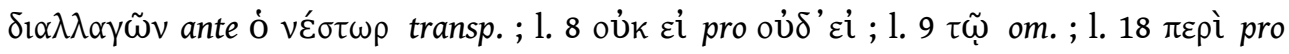

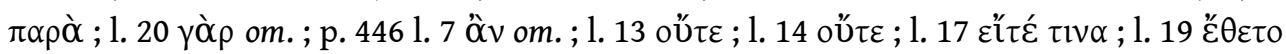

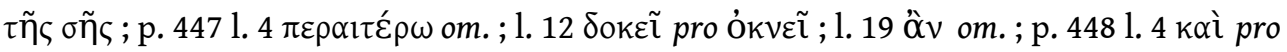

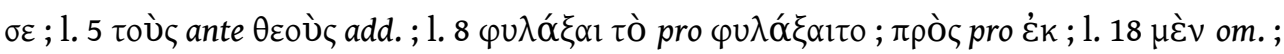

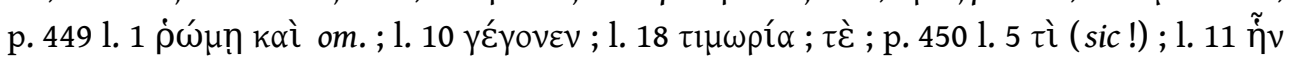

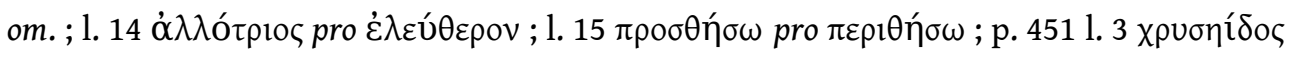

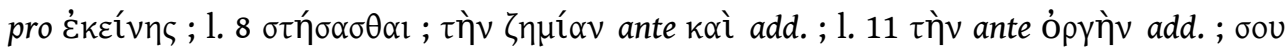

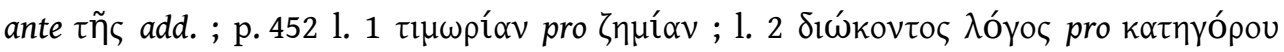

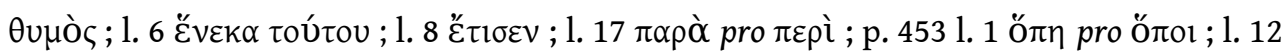

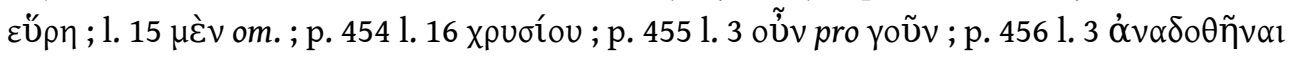

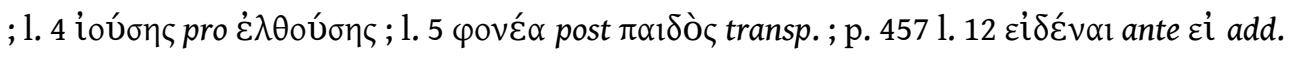

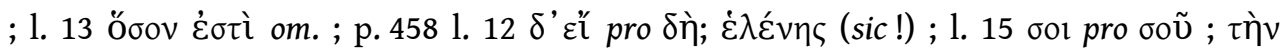

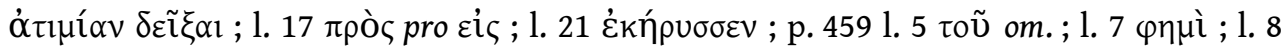

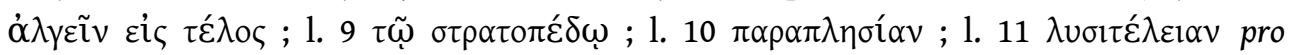

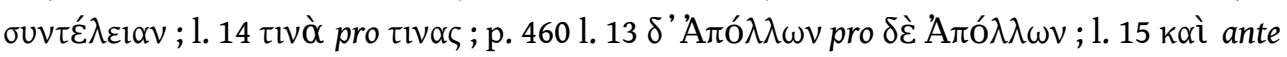




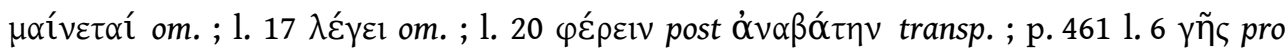

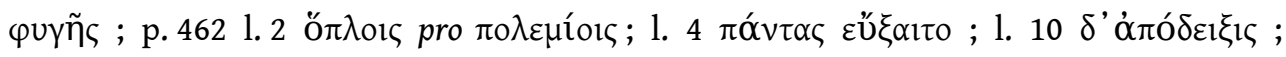

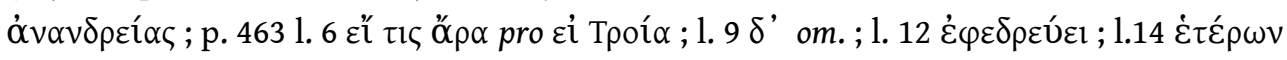

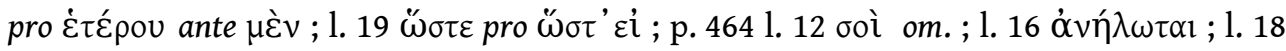

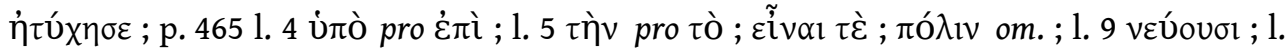

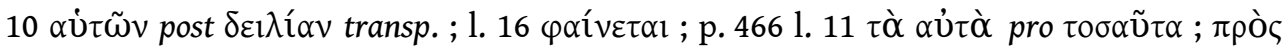

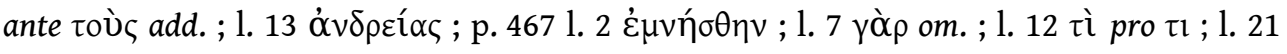

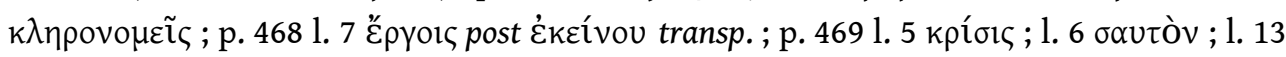

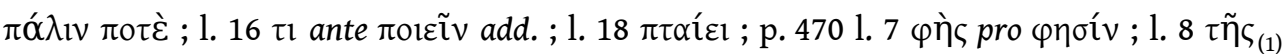

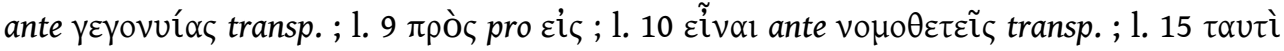

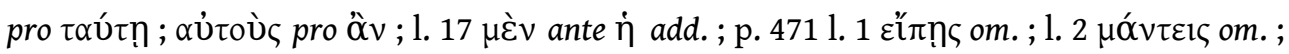

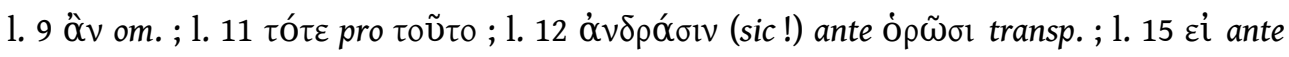

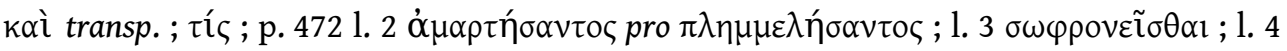

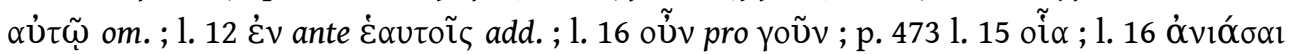

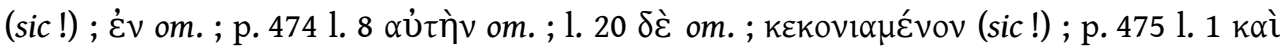

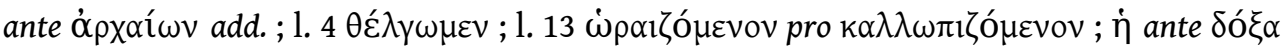
om. ; 1.20 tò om.

59. Morel 1606, f. $\mathrm{f}_{\mathrm{iii}} \mathrm{v}$ ([In Declamationes, Emendationes et Notata] Ad Patrocli suasoriam).

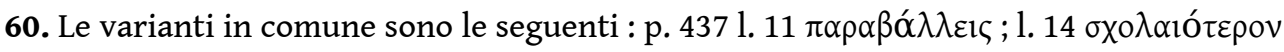

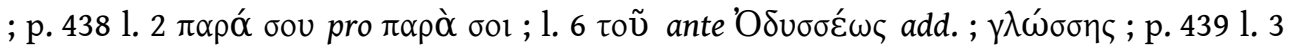

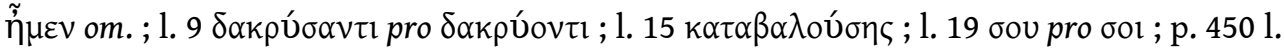

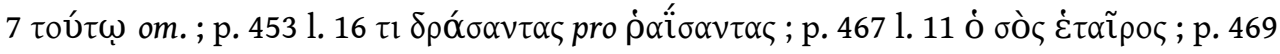

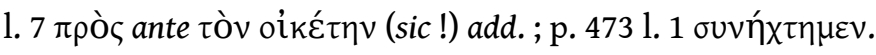

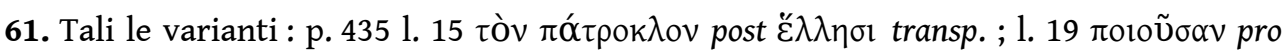

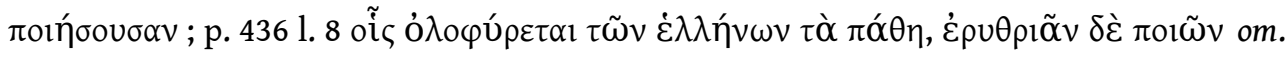

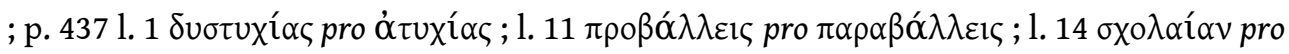

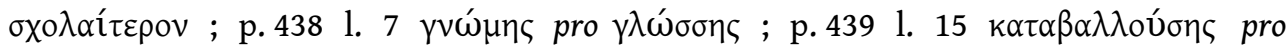

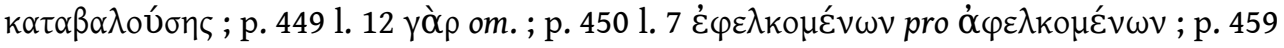

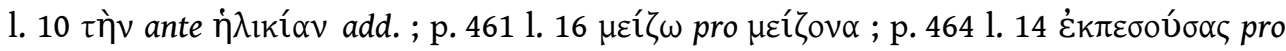

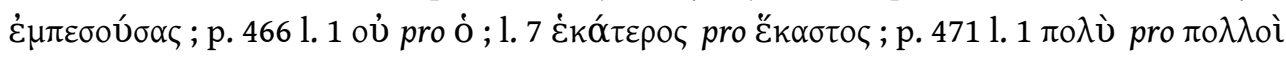

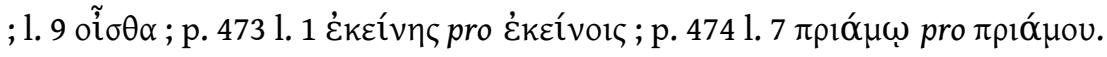

62. Il Par. gr. 2961, che risulta essere in assoluto il manoscritto con cui il testo del Morel ha più varianti in comune.

63. Ved. supra, n. 35.

64. Ved. D'Alessio 2016, 513-516; 520 [praes. 515].

65. Il manoscritto, del sec. XIV, è descritto in Lenz \& Behr 1976, LVII ; Foerster \& Richtsteig 1929, XXII, XXVIII ; Foerster 1909, 173-175.

66. Tra le lezioni in comune, si segnalano le seguenti : p. $4341.4 \tau \tilde{\omega} v$ ante $\dot{\varepsilon} \lambda \lambda \eta ́ v \omega v$ add.

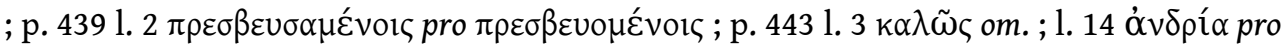

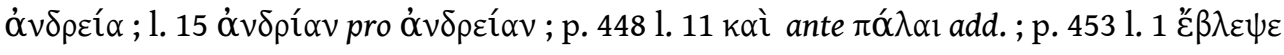

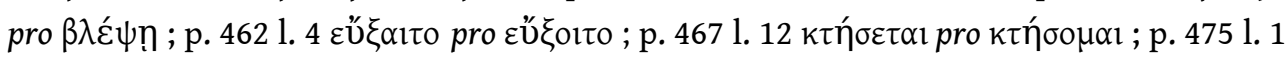

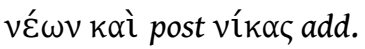

67. Per la descrizione del manoscritto, cartaceo del sec. XV, ved. Foerster 1903, 234 ; Foerster \& Richtsteig 1929, XXIV ; Omont 1883, 72. 
68. La dimostrazione della dipendenza diretta del Par. Suppl.gr. 656 dal Par. gr. 3018 sarà trattata in un mio prossimo lavoro.

69. Ved. Foerster 1903, 234.

70. Sulle missioni in Oriente di Minoïde Mynas, ved. Omont 1916 ; Dain 1957.

71. Ved. supra, n. 43.

\section{RIASSUNTI}

L'article vise à présenter, dans la première partie, deux manuscrits inédits de Chorikios, jusqu'ici inconnus des éditeurs. La seconde partie identifie les manuscrits utilisés par Frédéric Morel dans son édition du Patroclus (op. XXXVIII Foerster-Richtsteig) de 1606.

This paper aims to present, in the first part, two new manuscripts of Choricius, previously unknown by the editors. The second part identifies the manuscripts utilized by Frédéric Morel in his edition of the Patroclus (op. XXXVIII Foerster-Richtsteig) of 1606.

\section{INDICE}

Keywords : Choricius of Gaza, Patroclus (op. XXXVIII F. / R.), Rhetor (op. XLII F. / R.), manuscript tradition, Par. gr. 296, Par. gr. 2988, Par. gr. 3016, Par. gr. 3018, Esc. T II 9, Vat. gr. 224

Mots-clés : Chorikios de Gaza, Patroclus (op. XXXVIII F. / R.), Rhetor (op. XLII F. / R.), Morel Frédéric, tradition manuscrite, Par. gr. 296, Par. gr. 2988, Par. gr. 3016, Par. gr. 3018, Esc. T II 9 , Vat. gr. 224

\section{AUTORE}

\section{PAOLA D'ALESSIO}

EA 4276 L'AMo, Université de Nantes 